\title{
The detector for the far ultraviolet channel of the imaging instrument (FCU) on board the Spectrum-UV (WSO-UV) space telescope
}

Gómez de Castro, Ana I., Díez, Laura, Yañez, Javier, Prada, Iván, Araujo, Alejandra, et al.

Ana I. Gómez de Castro, Laura Díez, Javier Yañez, Iván Prada, Alejandra Araujo, Alejandra Salinas, Xavi LLamas, Mikhail Sachkov, Andrey Shugarov, "The detector for the far ultraviolet channel of the imaging instrument (FCU) on board the Spectrum-UV (WSO-UV) space telescope," Proc. SPIE 11444, Space Telescopes and Instrumentation 2020: Ultraviolet to Gamma Ray, 114446I (13 December 2020); doi: 10.1117/12.2560330 


\title{
The detector for the far ultraviolet channel of the imaging instrument on board the Spektr-UF (WSO-UV) space telescope
}

\author{
Gómez de Castro, Ana I. ${ }^{\mathrm{a}, \mathrm{b}}$, ${ }^{*}$ Díez, Laura ${ }^{\mathrm{c}}$, Yáñez, Javier ${ }^{\mathrm{a}, \mathrm{b}}$, Prada, Iván ${ }^{\mathrm{a}, \mathrm{b}}$, Araujo, \\ Alejandra $^{c}$, Salinas, Alejandra ${ }^{c}$, Llamas, Xavi ${ }^{c}$, Sachkov, Mikhail $^{\mathrm{a}, \mathrm{d}}$, Shugarov, Andrey ${ }^{\mathrm{a}, \mathrm{d}}$ \\ a Joint Center for Ultraviolet Astronomy, Universidad Complutense de Madrid, Edicio Fisac (Fac. EE Estadísticos), \\ Av. Puerta de Hierro s/n, Madrid, Spain, E-28040 \\ bAEGORA Research-Group, Facultad de CC. Matemáticas, Universidad Complutense de Madrid, Plaza de Ciencias \\ 3, Madrid, Spain, E-28040 \\ ${ }^{\mathrm{c}}$ SENER Aerospace, Aerospace Electromechanics Division, Creu Casas i Sicart, 86-88, Parc de l'Alba, Cerdanyola \\ del Vallès, Spain, E-08290 \\ ${ }^{\mathrm{d}}$ Institute of Astronomy of the Russian Academy of Sciences, Pyatniskaya St, 48, Moscow, Russia, 119017
}

\begin{abstract}
.
Spectr-UV/WSO-UV is the third and last of the Spektr missions (Spektr-Radio, Spektr-RG, Spektr-UF), spacebased observatories, of the Russian Scientific Space Program. Spektr-UF/WSO-UV is a 2-m class ultraviolet (UV) observatory to be set in geosynchronous orbit in 2025 from the Vostochny launching pad. The observatory is equipped with instrumentation for UV spectroscopy and imaging. The Universidad Complutense de Madrid together with Institute of Astronomy of the Russian Academy of Sciences are developing jointly the imaging instrument for the mission. The instrument has a UV-optical channel designed for wide field imaging from 174 to $600 \mathrm{~nm}$ and a far UV (FUV) channel for high resolution imaging in the 115-175 $\mathrm{nm}$ range. Also, some low dispersion spectroscopic capabilities are available in the FUV channel. In this contribution, the characteristics and architecture of the MCP detector being developed by Spain for the FUV channel are described.

The detector is a three steps detection system that transforms incoming UV photons into optical photons that are detected by a fast and configurable CMOS device. In a first step UV photons strike the photosensitive CsI substrate deposited on the MCP generating $\sim 1 \times 10^{5} e^{-}$per incoming photon. In a second step, the electrons pulse is transformed into a photon pulse at a phosphor (P46) interface; the output photons shower is captured by a bundle of optical fibers that transport the signal to the CMOS detector. In the final step, the optical photons are detected by the CMOS. Different algorithms are implemented to optimize photon counting for various purposes.
\end{abstract}

Keywords: ultraviolet astronomy, ultraviolet space observatories, ultraviolet detectors, photon counting detectors, MCP detectors, CMOS, events detection algorithms .

*Gómez de Castro, Ana I aig@ucm.es

\section{Introduction}

The MCP detector described in this work has been developed to be implemented in the far UV channel of the Field Camera Unit (FCU), the imaging instrument on board the space telescope Spektr-UF/WSO-UV; the project and the instrument development status are described in the contribution to this volume. ${ }^{1}$

The FCU is mounted on top of the optical bench, below the primary mirror of the T-170M telescope. The FCU has two channels, each fed by an independent pick off mirror. The near UV (NUV) channel is designed for wide field imaging from 174 to $600 \mathrm{~nm}$. The far UV (FUV) channel has capabilities for high resolution imaging in the 115-176 nm range. Also, some low dispersion spectroscopic capabilities around Lyman- $\alpha(\operatorname{Ly} \alpha)$ at $121.5 \mathrm{~nm}$ and the C IV resonance transition at $155.0 \mathrm{~nm}$ are available. Both channels are equipped with a set of filters to address a broad range of scientific goals. The instrument layout and the main features of the FCU are summarized in Figure 1 and Table 1, respectively. 
Table 1 Main features of the WSO-UV FCU.

\begin{tabular}{lll}
\hline Parameters & FUV channel & NUV channel \\
\hline Detector type & MCP & CCD \\
\hline Spectral range & $115-176 \mathrm{~nm}$ & $174-600 \mathrm{~nm}$ \\
\hline Effective area $\left(\mathrm{m}^{2}\right)$ & 0.23 at $122 \mathrm{~nm}$ & 1.1 at $255 \mathrm{~nm}$ \\
\hline Field of view & $1.40 \mathrm{arcmin}$ diameter & 7.5 arcmin diameter \\
\hline Scale & 0.041 arcsec/microchannel ${ }^{(*)}$ & 0.146 arcsec/pixel \\
\hline Final Image format & $2 \mathrm{k} \times 2 \mathrm{k}$ & $3 \mathrm{k} \times 3 \mathrm{k}$ \\
\hline No. of filters & Up to $10+2$ prims & Up to 15 \\
\hline${ }^{(*)}$ The micro-channels have an effective diameter of 12 microns (including the pitch)
\end{tabular}

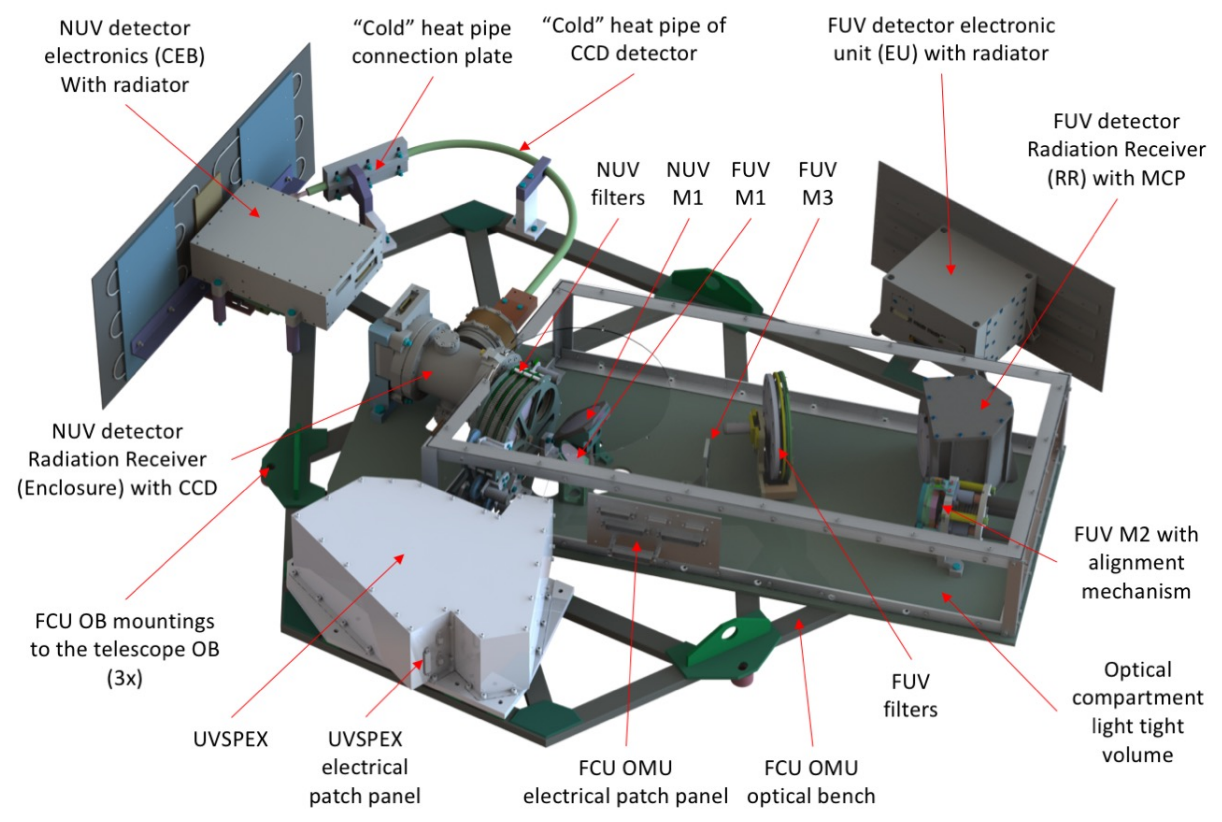

Fig 1 Layout of the FCU and location of the detectors.

FCU/FUV will support the following scientific operational modes:

- Imaging: The beam is focused on the selected channel (FUV) with no fast read-out

- Imaging Time-tag: Photon counting image mode on the detector. Photons will be count at a pace of at most $40 \mathrm{~ms} / \mathrm{read}-$ out over 5 minutes. Faster read-out is feasible in smaller "regions of interest" (ROIs).

- Spectroscopy: The beam is directed to the prisms. It may require refocusing the camera. 


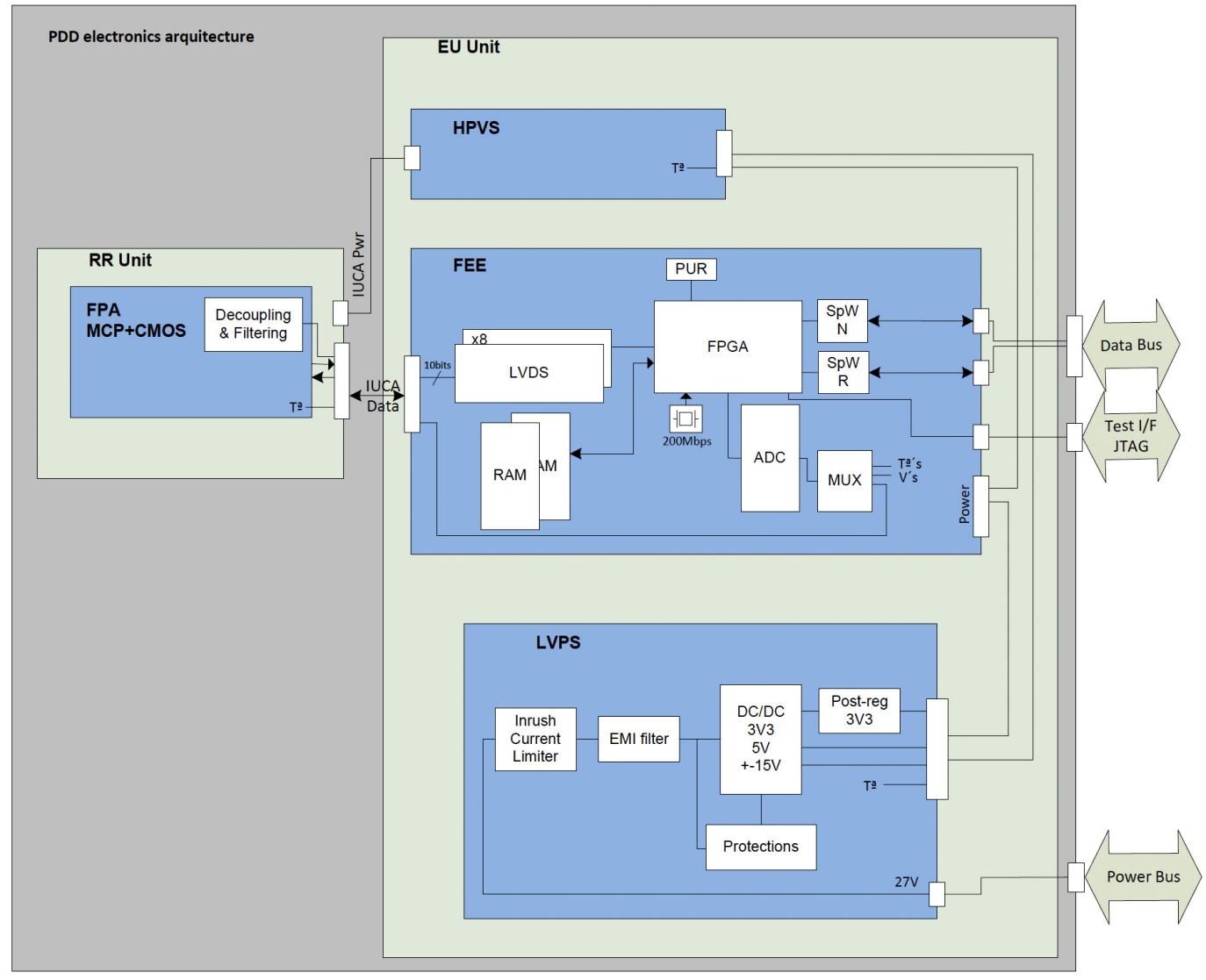

Fig 2 High Level Block Diagram of the detection device in the FCU/FUV channel

- Spectroscopy Time-Tag: Photon counting slitless spectroscopy mode on $30 \times 300$ pixels on the detector. Photons will be count at a pace of at most $40 \mathrm{~ms} / \mathrm{read}$-out over 5 minutes. This mode may require a previous acquisition image to set the target in a pre-defined area of fast read-out.

- Parallel-NUV: FCU/NUV Channel is used in parallel mode while using any other instrument.

The Photon Detection Device (PDD) of the FUV channel is composed by:

- Radiation Receiver (RR) for detection, amplification and conversion of FUV radiation into digital data. It comprises an MCP assembly and a CMOS detector coupled to the MCP.

- Electrical Unit (EU) for powering the RR, commanding the detector, acquiring and preprocessing the images and managing communications with the Camera Control Unit (CCU). It is composed by three boards, namely Low Voltage Power Supply (LVPS), the Front End Electronics (FEE) and the High Voltage Power Supply (HVPS).

- Inter Unit Cable Assembly (IUCA) Harness for data and power transmission between the RR and the EU. The high level block diagram of the PDD is depicted in Figure 2. 


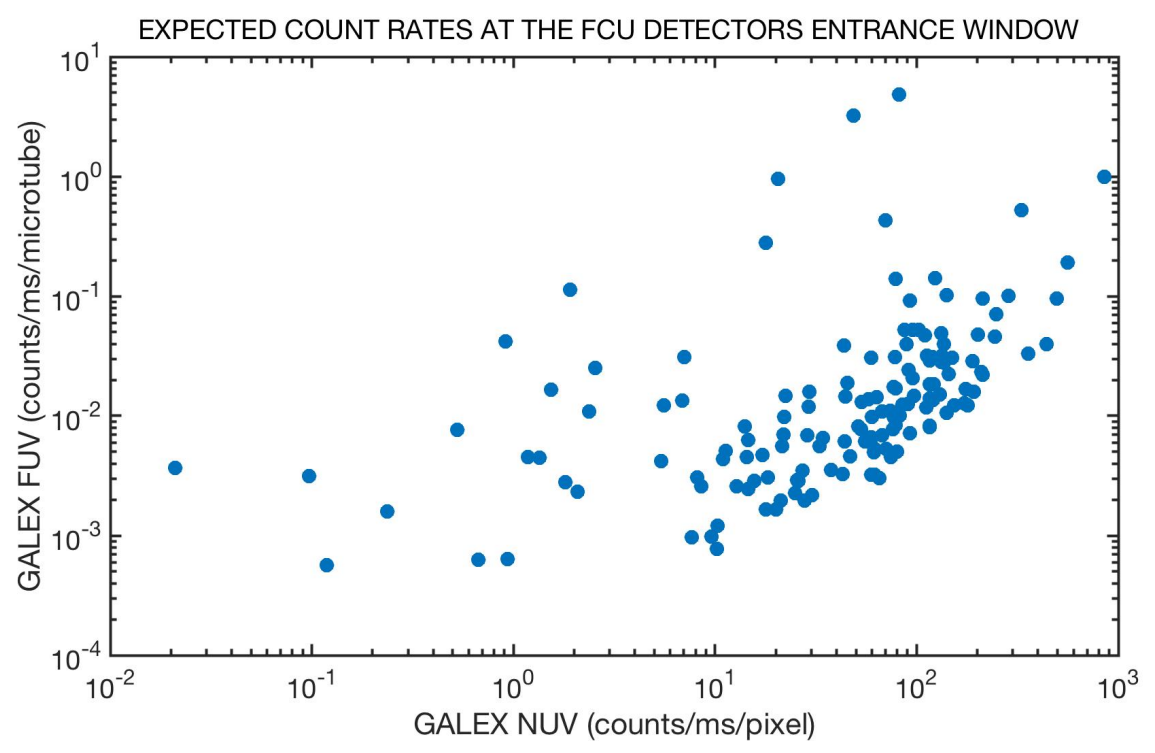

Fig 3 Expected count rate reaching the FCU detectors in the GALEX FUV and GALEX NUV photometric bands.

The detector is being manufactured by the Spanish company SENER Aeroespace under contract from the Ministry of Science and Innovation. In this work, the driving scientific requirements, the design and the state of development of the PDD are addressed. The scientific requirements are described in Section 2. The architecture of the RR and a comparison with similar MCP detectors flown in other astronomical missions is addressed in Section 3. The main operational modes are summarized in Section 4 together with the rationale behind the selection of the centroiding algorithms implemented for events detection. The current state of development is described in Section 5. A short summary is included at the end of the contribution (Section 6).

\section{Scientific requirements for the design of the detector}

The scientific requirements have been set to fulfill the needs of the most exigent scientific projects within the Spektr-UF/WSO-UV core program. ${ }^{1}$ The FUV channel is designed to resolve structures that could not be resolved with GALEX and to carry out slit-less spectroscopy of them on very specific spectral lines; Ly $\alpha$, OI and CI of special interest in exoplanetary research, $\mathrm{H}_{2}$ molecular bands in young planetary disks and CIV and HeII lines to measure the terminal velocities of massive early $\mathrm{B}$ and $\mathrm{O}$ stars in extragalactic star forming regions. Two especially demanding cases are described below.

\subsection{Monitoring exoplanets transits in Ly $\alpha$}

The expected Ly $\alpha$ count rate from exoplanet host stars can be derived in two manners.

A first estimate can be done from the observations of the Ly $\alpha$ profiles of solar-like stars carried with the Hubble Space Telescope. ${ }^{2}$ Taking into account the spectral dispersion of the prism and the effective area of the FCU/FUV channel, the total counts rate is not expected to exceed the 10 counts/s/pixel. However, these HST Ly $\alpha$ profiles are heavily absorbed by the interstellar medium and among the Spektr-UF/WSO-UV pre-approved core program proposals, it is the observation of high radial velocity cool stars, some of them presumably hosting exoplanets. In this case, the 


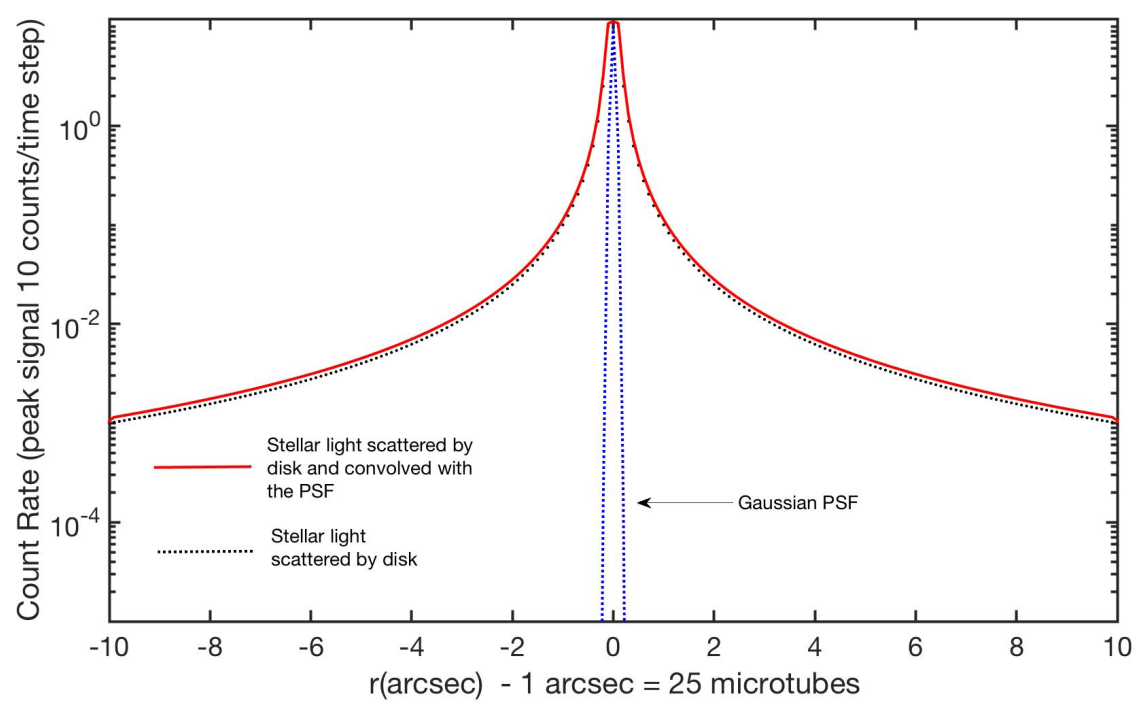

Fig 4 Scattered light profile from a debris disk; dust grains albedo is taken 1. Note that a non-realistic Gaussian PSF makes the wings of the stellar profile drop very rapidly. Dynamical ranges of $1 / 10^{3}$ are required to reach 5 arcsec.

Ly $\alpha$ flux may be significantly higher and the count rate may rise to 4 to 20 counts/pixel per 40 milliseconds. To get into the $<1$ count/pixel count-rate regime, photons should be read at an increased speed of 10 to 2 milliseconds. This velocity can be fulfilled if there is the possibility to read only small subframes.

GALEX observations of solar like stars can be used for an independent estimate of the expected Ly $\alpha$ count rates using their well known spectral energy distribution in the 115-200 nm range; typically the Ly $\alpha$ flux is 10-80 times the flux in the GALEX FUV range. We have searched the GALEX point sources catalogue for all known exoplanetary systems; most of them have been found by Kepler using the transit method. We have found 1,591 coincidences and, as shown in Figure 3, the total count rate in the GALEX FUV range is below 1 counts/microtube at the detector read-out frequency $(40 \mathrm{~ms})$. However, the Ly $\alpha$ is significantly stronger and moreover, cool stars are active flaring sources and the count rates may rise by a factor 10 during flares. Thus, a low gain, fast read-out mode in a small window needs to be implemented. Moreover, a dynamical range of $<1 / 10^{4}$ is required to detect the transits of large Earth-like exospheres and not just hot Jupiters. ${ }^{3}$

\subsection{Observation of faint disks around bright sources}

The detection of debris disks depends critically on the dynamical range of the detector. Typical sources for this type of studies are Herbig Ae/Be stars or F-type pre-main sequence (PMS) stars some of them very bright resulting in very high count rates (10 counts/40ms/pixel). The detection of the debris disk depends critically on the dynamical range but also on the optical PSF. If the PSF were purely Gaussian, the requirements in dynamical range would be significantly smaller, as shown in Figure 4.

\subsection{Summary requirements}

Two basic operation modes emerge from the requirements of the scientific programs: 
- Fast read-out in a tunable Region of Interest (ROI) to enable defining small regions on the detector (either in imaging or in spectroscopic mode) to be read as fast a $1 \mathrm{~ms}$. This provides a high dynamical range and enables the observation of transits in a wide range of sources (see Figure 3).

- Full frame observations that can be performed at a rate of $40 \mathrm{~ms}$. This provides a sensible mode of observation to image wide fields but with limitations in terms of dynamical range. A maximum threshold of 200,000 counts per second over the full frame is set for the MCP safety. This threshold is equivalent to a homogenous count rate of 0.047 counts/s/microtube, far above the FUV background that is $\sim 10^{-5}$ counts/s/microtube according to GALEX measurements. ${ }^{4}$

\section{RR: MCP detector architecture}

FCU/FUV detector is a three steps detection system that transforms incoming UV photons into optical photons that are detected by a fast and configurable CMOS device (see Figure 5). The opaque CsI photocathode is deposited directly on the face of the MCP; the QE of CsI is very low $(i i 0.1 \%)$ at wavelengths longer than $175 \mathrm{~nm}$ minimizing the contamination from Solar scattered light. Target photons strike the photocathode, releasing single photoelectrons that are amplified within the MCP, where a pulse of $\sim 1 \times 10^{5} e^{-}$is generated.

In a second step, the electrons pulse is transformed into a photon pulse at the phosphor (P46) interface; the output photons showers are captured by a bundle of optical fibers that transport the signal to the CMOS detector. An optical extender is used to couple the optical taper and the CMOS detector. A neutral density filter is be deposited on it (to avoid saturation on the CMOS detector).

The CMOS used is a CMV4000 from AMS, monochrome high speed CMOS image sensor with 2048 by 2048 pixels of $5.5 \mu \mathrm{m} \times 5.5 \mu \mathrm{m}$. The pixels are designed to achieve maximum sensitivity with low noise. Micro lenses are placed on top of the pixels for improved fill factor and quantum efficiency; up to $65 \%$ is reached at 530nm (phosphor emission). The CMOS is windowless for direct coupling by gluing it to the optical fiber.

The CMOS has sixteen 10bit digital LVDS serial outputs for providing the image, but only eight channels will be used. The image acquisition will be commanded via SPI interface. Only odd channels for the transmission will be used, which allow to un-power the even channels and to save power and read-out time.

The detection system has been optimized to increase the gain and preserve the image optical quality at the entrance of the detector. The effect in gain is also outlined in Figure 5; basically, a shower of 100,000 photons is generated at the MCP per UV photon at the entrance window. The main characteristics of the MCP detector are summarized in Table 2.

The MCP provision and the integration of the three components is carried by PHOTEK under contract from SENER-Aerospace.

At the Phosphor surface, the output photon shower has a typical FWHM of 60 microns. This is captured by the optical fibers of the fiber taper. The demagnification ratio is 3.62. Therefore, the shower is compressed into 16.8 microns (or 3 pixels) on the detector. The centroid of this shower is used to identify the microtube, on the MCP, where the UV photon has impacted. The section of the MCP is a hive of microtubes arranged in a hexagonal pattern. From end to end, the number of microtubes is $40 \mathrm{~mm} / 12 \mu \mathrm{m}=3,333$ micro tubes that project on the CMOS square grid made 

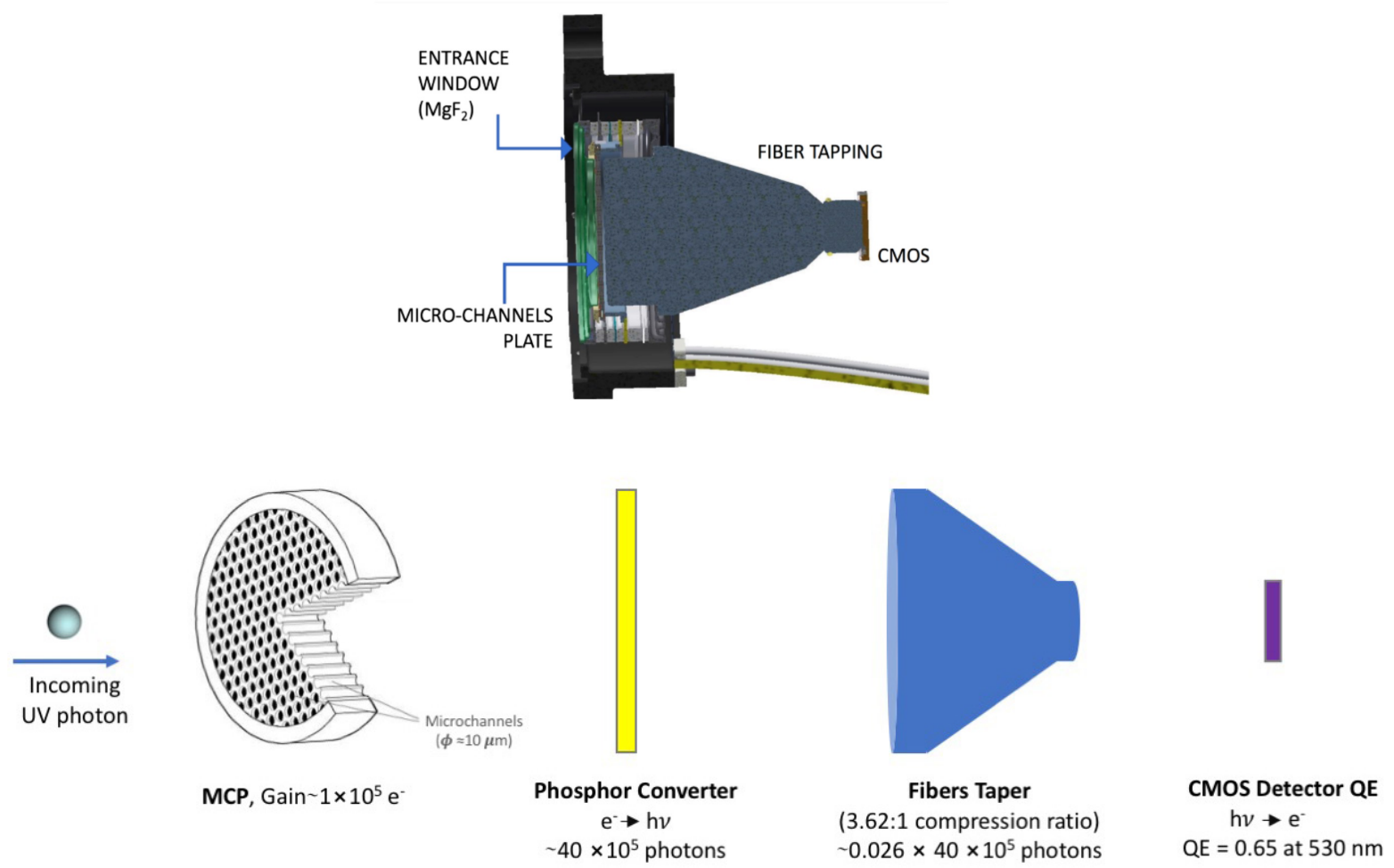

Fig 5 Top: sketch of the detector. Bottom: detector gain propagation.
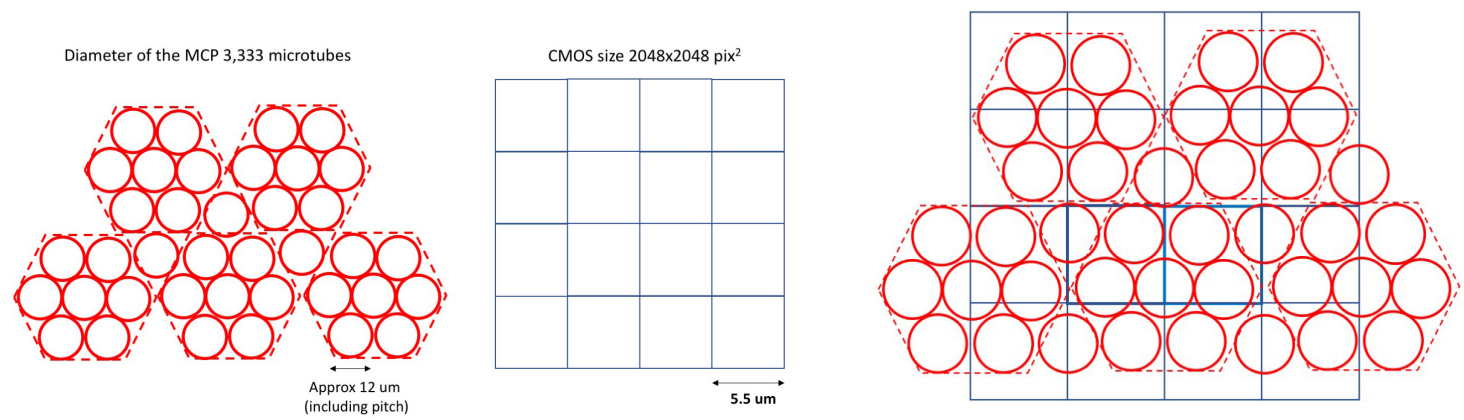

Fig 6 Representation of the projection of the MCP pores on the CMOS pixeled surface. On the left panel, the microtubes arrangement is outlined; each microtube has a projected section of $12 \mu \mathrm{m}$ because of the pitch. In the central panel, the CMOS read-out is shown; each CMOS pixel has a size of $5.5 \mu \mathrm{m}^{2}$. In the right panel, the end point of the optical fibres is projected on the CMOS surface; the projected diameter of the fibres is $3.3 \mu \mathrm{m}^{2}$

of 2048 pixels of $5.5 \mu \mathrm{m}$, as shown in Figure 6; 1.6 microtubes fit within a pixel in any given direction.

FCU/FUV detector design is based on the FUV detector implemented in the UltraViolet Imaging Telescope (UVIT) on Astrosat though performance in terms of sensitivity and read-out has been improved. A comparison between the FCU/FUV detector and other MCP detectors used in astronomical instruments is provided in Table 3. 
Table 2 Main features of the WSO-UV FCU.

\begin{tabular}{lll}
\hline CHARACTERISTICS & & \\
\hline Spectral range & & $115 \mathrm{~nm}-176 \mathrm{~nm}$ \\
\hline Diameter of the photosensitive area & & $40 \mathrm{~mm}$ \\
\hline Pore diameter on MCP & & $10 \mu \mathrm{m}$ \\
\hline Pore pitch width on MCP & & $12 \mu \mathrm{m}$ \\
\hline Optical tapper compression factor & size (pixels) & $3.62: 1$ \\
\hline CMOS detector characteristics & Pixel size & $2048 \times 2048$ \\
& Read out speed, full frame $(10$ bits $)$ & $180 \mathrm{fps}$ \\
& Read out speed, full frame $(12 \mathrm{bits})$ & $37 \mathrm{fps}$ \\
& ROI window capability & up to 8 \\
& Full well charge & $13,500 \mathrm{e}^{-}$ \\
& Dark current $(25 \mathrm{C})$ & 125 \\
\hline Saturation Count Rate & Dynamic range & $60 \mathrm{~dB}$ \\
\hline INTEGRATED SYSTEM & & $25 \mathrm{fps}$ \\
Maximum frame rate in full frame & mode & up to $1,000 \mathrm{fps}$ \\
Minimum frame rate in ROI mode & & $60 \mathrm{~dB}$ \\
& & 200,000 \\
\hline
\end{tabular}

\section{Operational modes and centroiding algorithms}

The detector operates in four possible modes:

- Accumulation Digital mode: images in FF (full frame) or ROI (region of interest) are digitally accumulated without further on-board processing (no algorithm implementation).

- Accumulation Analog mode: images with a maximum configurable ROI equivalent to $1024 \times 1024$ pixels are acquired. Event centroiding is performed on-board for event detection in each acquired image. Only pixel coordinates plus event counter are temporarily stored on-board and sent to ground. Both 7-cross and 5-square algorithms are available for this mode.

- Timetag 7-cross mode: each event is detected, time-tagged and sent to ground in an events file. The 7-cross algorithm is executed on-board to calculate the location of the event; event pixel coordinates and event value are calculated and sent.

- Timetag 5-square mode: events are detected using a 5-square algorithm that it is executed to calculate the impact position; event pixel coordinates, event value and $5 \times 5$ box pixel values around the event are sent. 
Table 3 Comparison between FCU/FUV detector and other MCP detectors used in UV instruments.

\begin{tabular}{lllll}
\hline Parameter & FCU/FUV & UVIT & OM & GALEX \\
\hline MCP & $\begin{array}{l}2 \text { stacks } \\
\text { (with chevron) }\end{array}$ & $\begin{array}{l}2 \text { stacks } \\
\text { (with chevron) }\end{array}$ & & $\begin{array}{l}3 \text { stacks } \\
\text { (Z-stack) }\end{array}$ \\
\hline Pore pitch & $12 \mu \mathrm{m}$ & $12 \mu \mathrm{m}$ & $12 \mu \mathrm{m}$ (MCP2) & $12 \mu \mathrm{m}$ \\
\hline Optical PSF (FWHM) & 2.5 pores & 2 pores & N/A & N/A \\
\hline FWHM electron shower & $60 \mu \mathrm{m}$ & $100 \mu \mathrm{m}$ & N/A & N/A \\
\hline Coupling with Optical Fibers & YES & YES & YES & NO \\
\hline Compression Factor & 3.62 & 3.33 & 3.37 & \\
\hline Read out & $\mathrm{CMOS}$ & $\mathrm{CMOS}$ & $\mathrm{CCD}$ & \\
\hline Read out pixel size & $5.5 \mu \mathrm{m}$ & $22 \mu \mathrm{m}$ & $8.86 \mu \mathrm{m}$ & \\
\hline Full frame read out speed & $25 \mathrm{~Hz}$ & $29 \mathrm{~Hz}$ & $2 \mathrm{~Hz}$ & \\
\hline
\end{tabular}

Time-tagged acquisition modes are available for a reduced area on the detector and with a scalable time resolution, $\tau$, given by,

$$
\tau(\mathrm{s})=\frac{25}{2048 \times 2048} \times \mathrm{N}_{\mathrm{ROI}}\left(\mathrm{pix}^{2}\right)=5.96 \times 10^{-6} \times \mathrm{N}_{\mathrm{ROI}}\left(\mathrm{pix}^{2}\right)
$$

with $\mathrm{N}_{\mathrm{ROI}}$ the total number of pixels in the ROI. It is foreseen that the detector will mainly operate in Accumulation Analog mode and Timetag 5-square mode.

Events processing is divided into two steps: detection and centroid calculation. Events are detected by three methods:

- Comparison with neighbours: one pixel whose amplitude is greater than that of its 24 surrounding neighbours ( 5 by 5 pixels box).

- Background threshold: The pixel amplitude is greater than a defined threshold level.

- Hot pixel and cosmic ray threshold: the absolute value of the maximum of the surrounding pixels minus the central pixel must be greater than a given threshold value. This criterion rejects well cosmic ray and hot/damaged pixels; it enables detecting big jumps between nearby pixels. This applies for any direction or similar values of nearby pixels, as expected in a cosmic ray strike.

The selection process is illustrated in Figure 7.

For the selection of the optimal centroiding algorithm, a computer based simulator has been developed firstly coded into MatLab and later re-coded in Python. ${ }^{5}$ Simple algorithms ${ }^{6}$ have been tested for linearity: 3-, 5-, 7-cross and 3-, 5-square, and 5-circle. Several photons are generated simultaneously with a statistical distribution that mimics the response of the MCP as measured 
A1

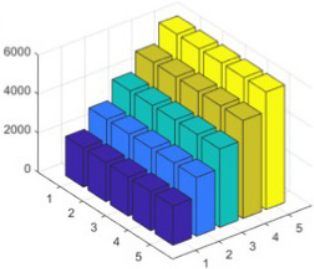

B1

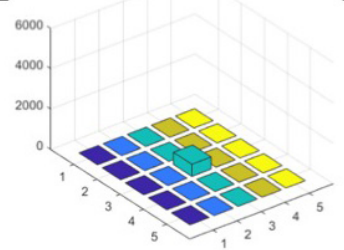

C1

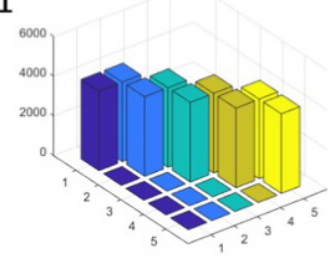

A2

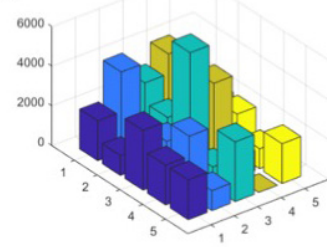

B2

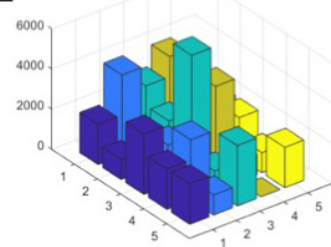

$\mathrm{C} 2$

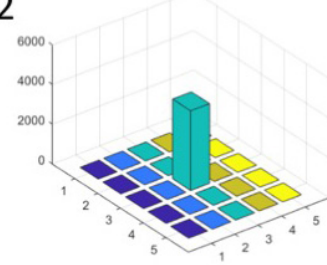

Fig 7 Illustration of the event detection criteria. According to the criterium of comparison with neighbours A1 is not an event and A2 it is. According to the use of a background threshold B1 is not an event and B2 it is. Events in C1 and $\mathrm{C} 2$ represent a cosmic ray and a hot pixel. Both are rejected by the third criterium.

in the laboratory as a result some of events (photons) may overlap. Then, we have defined an optimization factor, $\chi$, such that,

$$
\chi=\frac{\mid \text { SimulatedEvents }- \text { DetectedEvents } \mid}{\text { SimulatedEvents }}
$$

and tested for various background threshold levels. The results are shown in Figure 8. Clearly, the 5 -square algorithm is the best (for any background threshold). This algorithm is also the best to produce smooth flatfields ${ }^{7}$ though it is affected by saturation for bright objects and backgrounds as shown in the figure. Subpixel centroiding is required for some applications and gaussian centroiding is the best for this purpose however it is difficult to implement in the flight FPGA. The 7-cross algorithm is also good for this purpose and has been implemented for this reason.

The 5-square algorithm is the default for event centroiding in the FCU/FUV channel. It will be used by the on-board software to generate the raw list of events that will be sent to the ground as well as to produce the accumulated images in the Accumulation Analog mode. Moreover, the values of the 24 pixels around any event will also be sent to ground to enable post-processing (for instance, using a gaussian algorithm).

\section{State of development}

The PDD has already gone through the Critical Design Review (CDR) and the engineering model is being manufactured. The validation of the engineering model will be completed during the 


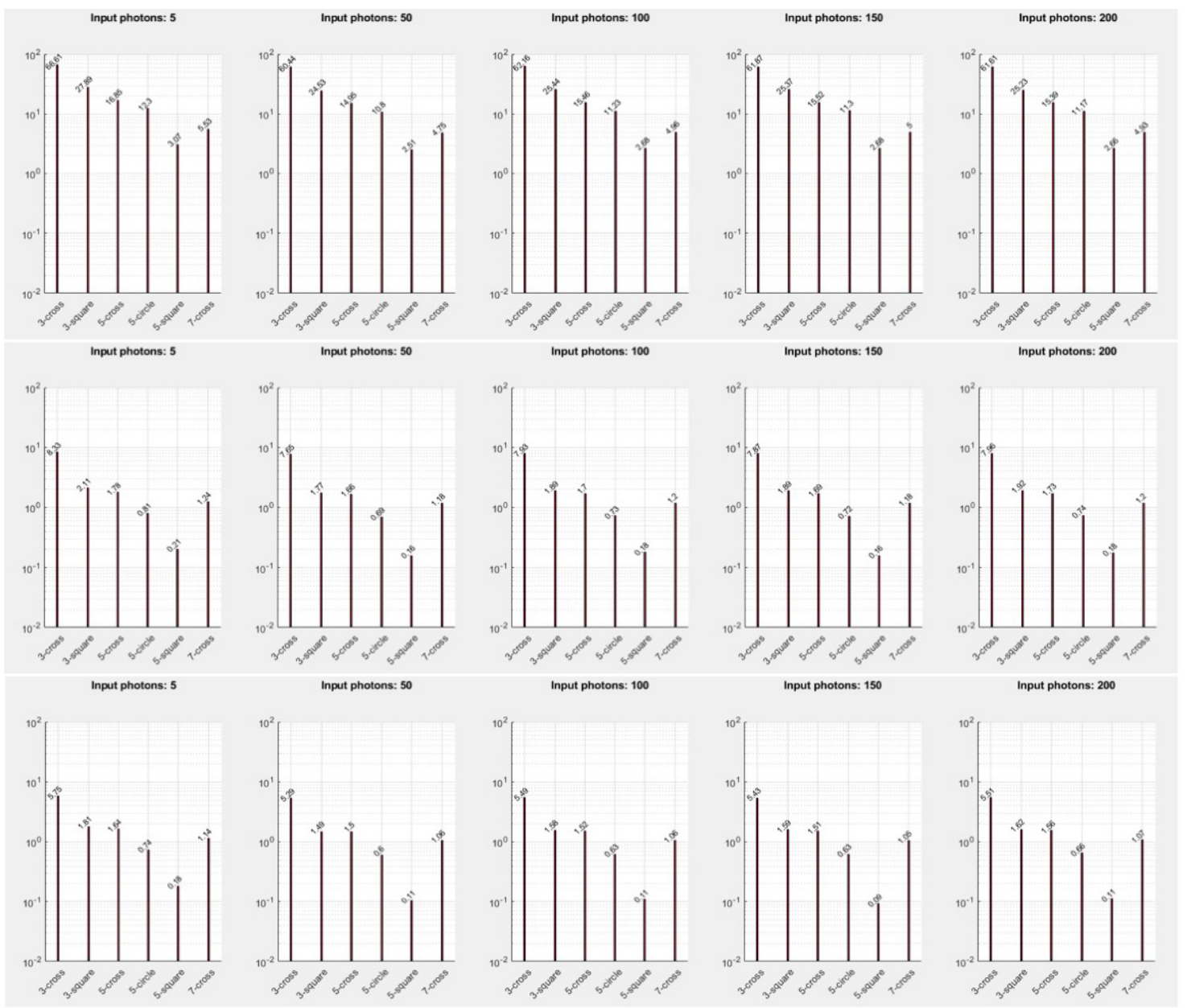

Fig $8100 \times \chi$ is represented in each sub-panel of the figure for the 6 algorithms tested and several conditions. From left to right, the number of simultaneous simulated photons increases from 5 to 200 . In the top panel, no threshold level is set for event detection. In the middle, the level is 30 and in the bottom 60. 5-square, 5-circle and 7-square provide the best results. 


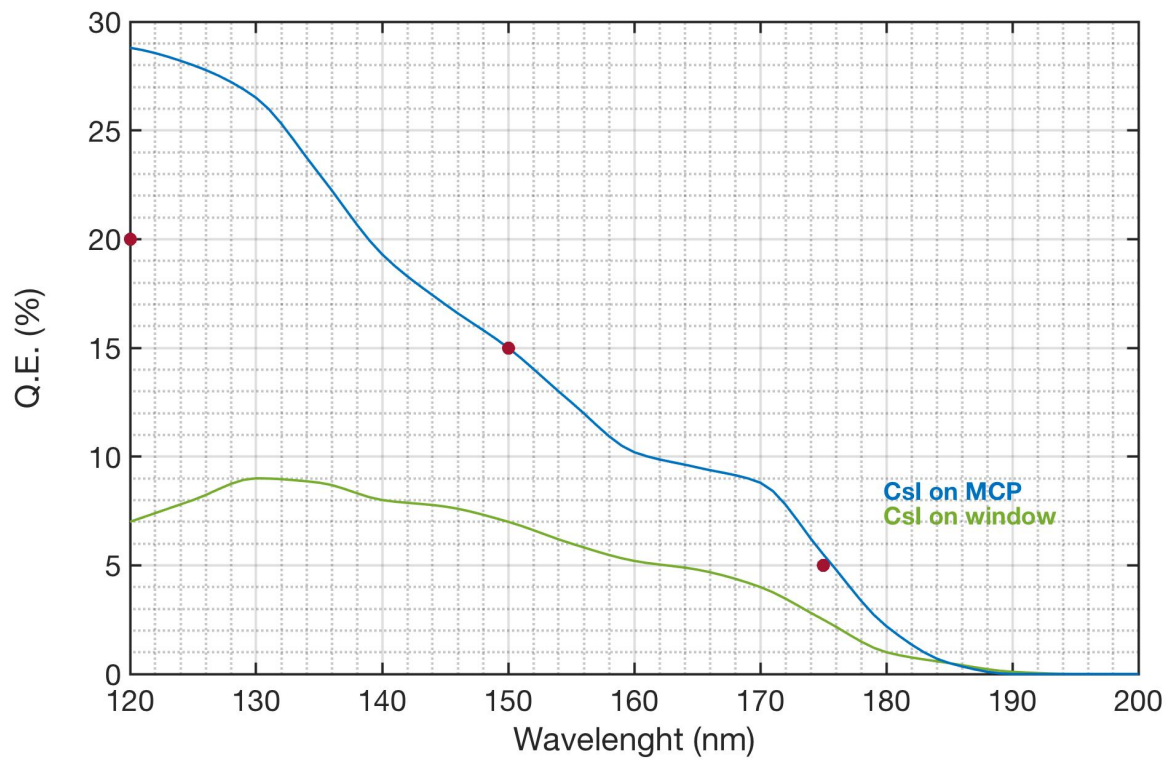

Fig 9 QE of the RR compared with standard values for CsI substrate deposited on the shielding window. Measurements are shown before encapsulation. After encapsulation, the absorbance of the $\mathrm{MgF}_{2}$ window reduces the $\mathrm{QE}$ to $20 \%$ at $121 \mathrm{~nm}$.

first months of 2021 and the flight model produced just afterward, to be sent to the Institute of Astronomy of the Russian Academy of Sciences before the end of 2021 for its integration in the instrument.

The most novel features in the PPD relate with the deposit of the CsI substrate and the FPGA.

To enhance the QE of the detector, the CsI substrate has been deposited at the entry face of the MCP micropores instead of the most conventional deposit on the sealing window. The qualification campaigns are showing that $\mathrm{QE}$ at $121 \mathrm{~nm}$ (Ly $\alpha$ wavelength) reaches 20\%, which represents a factor of $\sim 3$ improvement in sensitivity. The deposit has been carried out by the team in the University of Leicester under contract from PHOTEK (see Figure 9).

The control FPGA is implemented in a radiation hardened device from NanoXplore developed, at European level, to break the dependence from US in this key component for deep space missions. The NanoXplore device has a capability of, at least, 4,400,000 gates and an embedded memory. This memory can be configured as standard SRAM or as FIFO memories. The SRAM space are inherently susceptible to upsets caused by high-energy particles encountered in space, then the memory structures implemented internally into the FPGA will be protected by EDAC. The control FPGA is in charge of the sensor commanding, readout and acquisition signals generation and CCU interfacing. A $200 \mathrm{MHz}$ integrated oscillator shall output the clock signal which will be connected to the control FPGA input clock.

The images acquired by the FPGA will be sent directly to the CCU, but if the accumulation observation mode is used, it is required to stored temporarily a full-frame image (4 Mpixel) locally on-board. During accumulation observation mode, only photon position is saved in the pixel position where it has been detected. In subsequent readout, if an impact is detected, it will be added to the pixel where it has been produced. The resolution for counting impacts is 16 bits for each pixel, which means that up to 65535 impacts, can be counted. 


\section{Closing remarks}

The development of the PPD for the FCU/FUV channel is a four year project ending in 2021, on time for the integration of the FCU. Spektr-UF/WSO-UV will be the first 2-m class telescope equipped with instrumentation for imaging in the 115-176 nm range high above the geo-corona in a dark FUV sky. The FCU/FUV channel is a equipped with a sensitive and versatile detector that will optimize the scientific return from the mission.

\section{Acknowledgments}

This work has been developed under contract of the Ministry of Industry, Commerce and Tourism of Spain and research grant ESP 87813-R from the Ministry of Science and Innovation of Spain.

\section{References}

[1] M. Sachkov, B. Shustov and A. I. Gómez de Castro, "The World Space Observatory - Ultraviolet mission: science program and status report", Proc. SPIE, in press, (2020).

[2] A. Youngblood, K. France, Parke R.O. Loyd, et al., "The MUSCLES Treasury Survey. II. Intrinsic LY and Extreme Ultraviolet Spectra of K and M Dwarfs with Exoplanets", Astrophysical Journal, vol. 824, id. 101 (2016)

[3] A.I. Gómez de Castro, L. Beitia-Antero and S. Ustamujic, "On the feasibility of studying the exospheres of Earth-like exoplanets by Lyman- $\alpha$ monitoring. Detectability constraints for nearby M stars", Experimental Astronomy, vol. 45, issue 2, 147-163, (2018).

[4] M. Armengot and A. I. Gómez de Castro, "Signatures of diffuse interstellar gas in the Galaxy Evolution Explorer all-sky survey", Astronomy and Astrophysics, vol. 631, id.A59, 8 pp. (2019).

[5] I. Prada, A. I. Gómez de Castro and J. Yáñez, "MCPSim-Py: an open source python-based simulator of the performance of MCP photon-counting detectors", Proc. SPIE, in press, (2020).

[6] K. Suhling, R. Airey and B. Morgan, "Optimisation of centroiding algorithms for photon event counting imaging", Nuclear Instruments and Methods in Physics Research, Section A: Accelerators, Spectrometers, Detectors and Associated Equipment, vol. 437, no. 2-3, pp. 393-418, (1999).

[7] J. Hutchings, J. Postma, D. Asquin and D. Leahy, "Photon event centroiding with UV photoncounting detectors", Publications of the Astronomical Society of the Pacific, vol. 119, no. 860, pp. 1152-1162, (2007).

\section{List of Figures}

1 Layout of the FCU and location of the detectors.

2 High Level Block Diagram of the detection device in the FCU/FUV channel

3 Expected count rate reaching the FCU detectors in the GALEX FUV and GALEX NUV photometric bands.

4 Scattered light profile from a debris disk; dust grains albedo is taken 1. Note that a non-realistic Gaussian PSF makes the wings of the stellar profile drop very rapidly. Dynamical ranges of $1 / 10^{3}$ are required to reach 5 arcsec. 
5 Top: sketch of the detector. Bottom: detector gain propagation.

6 Representation of the projection of the MCP pores on the CMOS pixeled surface. On the left panel, the microtubes arrangement is outlined; each microtube has a projected section of $12 \mu \mathrm{m}$ because of the pitch. In the central panel, the CMOS read-out is shown; each CMOS pixel has a size of $5.5 \mu \mathrm{m}^{2}$. In the right panel, the end point of the optical fibres is projected on the CMOS surface; the projected diameter of the fibres is $3.3 \mu \mathrm{m}^{2}$

7 Illustration of the event detection criteria. According to the criterium of comparison with neighbours A1 is not an event and A2 it is. According to the use of a background threshold B1 is not an event and B2 it is. Events in $\mathrm{C} 1$ and $\mathrm{C} 2$ represent a cosmic ray and a hot pixel. Both are rejected by the third criterium.

$8100 \times \chi$ is represented in each sub-panel of the figure for the 6 algorithms tested and several conditions. From left to right, the number of simultaneous simulated photons increases from 5 to 200. In the top panel, no threshold level is set for event detection. In the middle, the level is 30 and in the bottom 60. 5-square, 5-circle and 7-square provide the best results.

9 QE of the RR compared with standard values for CsI substrate deposited on the shielding window. Measurements are shown before encapsulation. After encapsulation, the absorbance of the $\mathrm{MgF}_{2}$ window reduces the QE to $20 \%$ at $121 \mathrm{~nm}$.

\section{List of Tables}

1 Main features of the WSO-UV FCU.

2 Main features of the WSO-UV FCU.

3 Comparison between FCU/FUV detector and other MCP detectors used in UV instruments. 